Systematic Review/Meta-Analysis

\title{
The Efficacy and Treatment Fidelity of Kinesiology Taping in Conjunction With Conservative Treatment Interventions Among Individuals With Shoulder Pain: A Systematic Review with Meta- Analysis
}

Paul A Salamh, PT, DPT, PhD ${ }^{1}$, William J Hanney, PT, PhD ${ }^{2}{ }^{\text {a }}$ Christopher S Cory, PT, DPT ${ }^{3}$, Haley E Condon, $\mathrm{PT}$, DPT ${ }^{4}$ Xinliang Liu, PhD ${ }^{5}$, Morey J Kolber, PT, PhD ${ }^{6}$

${ }^{1}$ Krannert School of Physical Therapy, University of Indianapolis, 2 School of Kinesiology and Physical Therapy, University of Central Florida, 3 Franciscan Health, ${ }^{4}$ Select Physical Therapy, ${ }^{5}$ Department of Health Management and Informatics, University of Central Florida, 6 Department of Physical Therapy, Nova Southeastern University

Keywords: conservative care, intervention, kinesiology taping, treatment fidelity, shoulder pain

https://doi.org/10.26603/001c.24251

International Journal of Sports Physical Therapy

Vol. 16, Issue 3, 2021

\section{Purpose}

Kinesiology taping is a common intervention used to treat individuals with shoulder pain. While there have been several studies published to date evaluating the effectiveness of this intervention, a systematic review with meta-analysis synthesizing the collective effectiveness of kinesiology taping is not available. Therefore, the purpose of this study was to perform a systematic review with meta-analysis investigating the efficacy and treatment fidelity of kinesiology taping (KT) in combination with conservative interventions for shoulder pain.

\section{Methods}

Databases (PubMed, EMBASE, SportDiscus, CINAHL) of studies published in English meeting criteria were searched to October 2019. Methodologic quality was assessed utilizing the Modified Downs and Black checklist. Treatment fidelity was evaluated using a modified fidelity tool. Random effects meta-analyses were performed when an outcome (disability, pain, range of motion (ROM)) was reported by two or more studies. Overall effect size (pooled random effects) was estimated for studies with acceptable clinical homogeneity.

\section{Results}

When KT was used with conservative treatments, meta-analysis revealed large effect sizes for improvements in disability (standard mean difference (SMD) $=-1.35 ; 95 \%$ CI, -2.09 to -0.60 ) and ROM (SMD = 0.96; 95\% CI, 0.60-1.33) with no significant effects for pain. The average Modified Downs \& Black score for bias was $11.5 \pm 3.9$. Of 10 retained studies, only two had good treatment fidelity.

\section{Conclusions}

Adding KT to interventions performed in clinical settings appears to demonstrate efficacy regarding disability and ROM when compared to conservative interventions alone.

However, despite reasonably good methodologic quality, fidelity was lacking in a majority of studies. Because of its impact on the implementation of evidence-based practice, lower fidelity should be considered when interpreting results.

\footnotetext{
a Corresponding author:

William J. Hanney

School of Kinesiology and Physical Therapy

University of Central Florida; 12805 Pegasus Drive \#262, Orlando, FL 32816-2205

william.j.hanney@ucf.edu
} 


\section{INTRODUCTION}

Shoulder pain affects up to $67 \%$ of the adult population throughout their lifespan. 1,2 The etiology of shoulder pain is multifactorial and inclusive of various impairments, including but not limited to restricted range of motion (ROM), pain, decreased strength, and impaired motor coordination. ${ }^{3-5}$ A variety of interventions are utilized in a multimodal approach for treating shoulder pain and dysfunction with the primary focus on restoring pain free shoulder mobility and mitigating ensuing activity restrictions. ${ }^{5-7}$ In recent years, kinesiology taping (KT) procedures have become increasingly popular. The proposed benefits of KT include providing a tactile cue via the skin to assist or limit movements, modulate pain, and increase microcirculatory flow which is thought to assist in reducing inflammation. ${ }^{8-12} \mathrm{Ki}$ nesiology tape refers to various types of elastic adhesive materials applied to joints in order to achieve various therapeutic effects. ${ }^{13,14}$ In particular, KT is widely utilized to treat impairments of the shoulder due to ease of accessibility and the ability to adapt to various body morphologies.

Current evidence investigating the effects of KT on shoulder movement and overall function among both asymptomatic and symptomatic individuals has been contradictory. Some studies have concluded that KT assists in improving shoulder joint mechanics and functional movement, while others suggest KT has no impact on joint position sense and in some cases can even negatively impair joint position sense. ${ }^{9,14-18}$ Furthermore, KT may impair muscular performance and not be well-tolerated by some individuals. ${ }^{12}$ Results from other studies have demonstrated that KT has no significant difference when compared to placebo treatment, manual therapy, or different types of tape. ${ }^{12,13}$ This contradictory evidence makes it difficult to determine the clinical utility of KT for those individuals with shoulder disorders seeking conservative treatment options. Additionally, the treatment fidelity associated with studies investigating the efficacy of KT has not been investigated but is a requisite for the implementation of evidence based clinical practice. Treatment fidelity is utilized in order to ensure that the particular intervention being studied is carried as described in the original protocol of an investigation. Without treatment fidelity it is uncertain as to whether those providing the intervention did so in the same manner each time and also makes it difficult to recreate similar investigations in the future.

Currently, systematic reviews have attempted to synthesize the literature surrounding KT. However, these systematic reviews either focus on musculoskeletal conditions as a whole or a specific shoulder pathology such as neurological conditions and rotator cuff tendinopathy. ${ }^{18-20}$ A recent systematic review sought to investigate the impact of KT in combination with a therapeutic intervention but focused solely on subacromial pain syndrome (SAPS) and retained only 4 studies which did not allow for any meaningful conclusion to be drawn. ${ }^{21}$

Given that the combination of applying KT in addition to traditional physical therapy intervention closely mirrors how KT is utilized in a majority of clinic settings, it would appear most relevant to investigate this relationship if our analysis is to offer clinical utility. ${ }^{22-25}$ Thus, the purpose of this study was to perform a systematic review with metaanalysis investigating the efficacy and treatment fidelity of $\mathrm{KT}$ in combination with conservative interventions for shoulder pain. Multi-modal interventions were considered for inclusion as a means of supporting external validity and clinical impact.

\section{METHODS}

\section{GUIDELINES}

This systematic review used the Preferred Reporting Items for Systematic Reviews and Meta-Analyses (PRISMA) guidelines during the search and reporting phase of the research process. The PRISMA 2009 statement includes a 27-item checklist developed to improve reporting of systematic reviews and meta-analyses. ${ }^{26}$

\section{LITERATURE SEARCH}

An online literature search was conducted utilizing PubMed, EMBASE, SportDiscus and CINAHL from the dates of their origin until October 2019. The search strategy was created and performed by a biomedical librarian. An example of the search strategy used for the PubMed database can be seen in Supplement 1 and similar strategies were utilized for the three remaining databases. This study was registered using the International Prospective Register of Systematic Reviews (PROSPERO) in July 2017 with the corresponding reference number CRD42017074147.

\section{STUDY SELECTION}

Appraisal of all titles was performed independently by two authors (CC) and (HC) after the initial online literature search. The abstracts of the retained titles were then reviewed to determine if inclusion criteria were met. Full-text versions of those studies whose abstracts met the inclusion criteria were accessed and read to determine their eligibility for the review. The same two authors undertook the study selection process throughout with discrepancies being decided by an independent author if necessary (no discrepancies were present). The inclusion criteria consisted of: (a) studies where KT was applied to the shoulder complex only; (b) studies including subjects diagnosed with shoulder pathology; (c) studies reporting objective measures of pain and function; and (d) studies comparing KT in conjunction with conservative treatment interventions. In addition, all study designs (including case studies) were included without restrictions on publication date or age of subjects, or duration of symptoms. Studies were excluded if (a) they were not published in the English language or (b) they had a mixed patient population (individuals with other orthopedic and neurological conditions).

\section{DATA EXTRACTION}

The data and results from each study selected for review was extracted using a standardized Population, Treatment, Comparison, Outcome and Setting (PICOS) format. ${ }^{27}$ This format includes the characteristics of the population studied, treatments performed, comparative treatments, pri- 
mary and secondary outcomes, and the setting in which the data was collected. Data was extracted, analyzed and reviewed by two authors (PS) and (HC). A single author (PS) extracted data and this was verified by a second author (HC). No discrepancies were identified between authors.

\section{METHODOLOGICAL QUALITY TOOL}

The Modified Downs and Black checklist was used to evaluate methodological quality within the individual studies. ${ }^{28}$ The Downs and Black checklist in its original format contains 26 items and has been shown to be a valid and reliable measure of methodological quality for randomized and nonrandomized studies. ${ }^{28}$ Given that various study designs were present among those articles retained for review, the authors chose to utilize a modified version of the Downs and Black checklist. The Modified Downs and Black checklist has been widely utilized to assess methodological quality. $^{29-32}$ Six sections are present as part of the modified checklist and include patient selection bias (items 1-4), comparison (item 5), outcomes (items 6-8), reporting findings/statistical analysis (items 9-11), confounding (12 \& 13), and power (items $14 \& 15$ ). Each item within the Downs and Black tool corresponds to a question and answered either yes $(\mathrm{Y})$, no $(\mathrm{N})$, or unable to determine $(\mathrm{U})$. The maximum score for the checklist is 16 with all individual items rated as either yes $(=1)$ or no/unable to determine $(=0)$ except for item 12 that may be rated as yes $(=2)$, partially $(=1)$, or no/unable to determine $(=0)$. Two authors (PS) and (CC) independently scored the retained articles for methodological quality. Discrepancies were handled through discussion to reach a consensus.

\section{TREATMENT FIDELITY ASSESSMENT}

Treatment fidelity assessments were performed to determine if the studies included in this review followed appropriate procedures to ensure that valid comparisons of replicable interventions were being executed. ${ }^{33-35}$ We chose to assess treatment fidelity by utilizing a modified version of a tool developed by Borelli et al. ${ }^{36}$ The original scale was appropriate for psychological interventions, which contained domains that are outside the scope of clinical interventions. A modified version of the tool was utilized to identify the 11 items that represented the essential items pertaining to a study investigating clinical interventions and not phycological interventions. Although this modified scale has not been validated, it has been utilized by others in similar reviews to examine treatment fidelity. ${ }^{37,38}$ Items are scored as 0 (not present), 1 (minimally described), or 2 (more than minimally described). Scores were recorded as both the summary (number present, maximum possible score 11) and total score (maximum possible 22) and were determined by a single author (PS), a researcher with a clinical background in musculoskeletal orthopaedics and experience in fidelity scoring We identified item summaries $\geq 6$ and total scores $\geq 12$ as studies that exhibited good treatment fidelity. These scores are based on prior investigations in this area and less likely to misrepresent the actual effect of the treatment provided in any given study. 38

\section{DATA SYNTHESIS AND ANALYSIS}

The mean and standard deviation in the outcome variables of pain, disability, and ROM preintervention and postintervention, as well as sample sizes for the experimental and control groups were extracted. The assessments administered at the end of the intervention or closest to one-month post intervention were used in the meta-analysis. Effect sizes based on standard mean differences (SMDs) were calculated for each outcome variable. When not provided, the standard deviation of the change score was computed from the standard deviations of the pre-post scores, using a correlation of $0.50 .{ }^{39}$ Studies were grouped by the comparison category (e.g., kinesiology taping plus exercise vs. exercise only). Random effects meta-analyses were performed for each subgroup of studies to examine an outcome variable when an outcome was reported by two or more studies. A pooled random-effects estimate of the overall effect size was estimated for all studies with acceptable clinical homogeneity. For pain and disability scales (the numeric pain rating scale (NPRS) and visual analog scale (VAS) for pain, Disability of Arm and Shoulder Questionnaire (DASH), and Shoulder Pain and Disability Index (SPADI)), higher scores indicate greater pain or disability. By contrast, higher scores reflect higher quality of shoulder function in the Constant score. Therefore, the direction of Constant score was reversed when pooled with other disability scales. For an increase in ROM, a positive effect size favors the experimental group over the control group. The effect size of the interventions was categorized into one of the three levels based on SMD $(<0.40=$ small, 0.40 to $0.70=$ moderate, $>0.70=$ large). ${ }^{27}$

The presence of heterogeneity was evaluated using the Q statistic after the meta-analysis. ${ }^{39,40}$ The Q statistic follows a chi-squared distribution with $n-1$ degrees of freedom. A small $p$-value $(p<0.05)$ for the $\mathrm{Q}$ statistic indicates that heterogeneity is present, and the meta-analysis model has some unaccounted-for bias. All meta-analyses were conducted in Stata (version 12.1) statistical software (StataCorp, College Station, TX) and the metan command. All data analysis was performed by (XL), an academician with a specialty in biostatistics and health informatics.

\section{RESULTS}

\section{SEARCH STRATEGY}

Database searches yielded 618 independent study titles, resulting in 357 after duplicates were removed. After a title and abstract search, 345 studies were removed because they did not meet the a priori inclusion/exclusion criteria. After review of the full-text studies, 10 were ultimately retained for analysis (Figure 1). ${ }^{22,23,25,41-47}$ Study characteristics for each of the 10 included studies are included in the PICOS table (Appendix A).

\section{METHODOLOGICAL QUALITY}

Methodological quality assessment revealed a range of values (Table 1). Of a 15 total item maximum and 16 total possible points, the average score was $11.5 \pm 3.9$ (median 12 , 


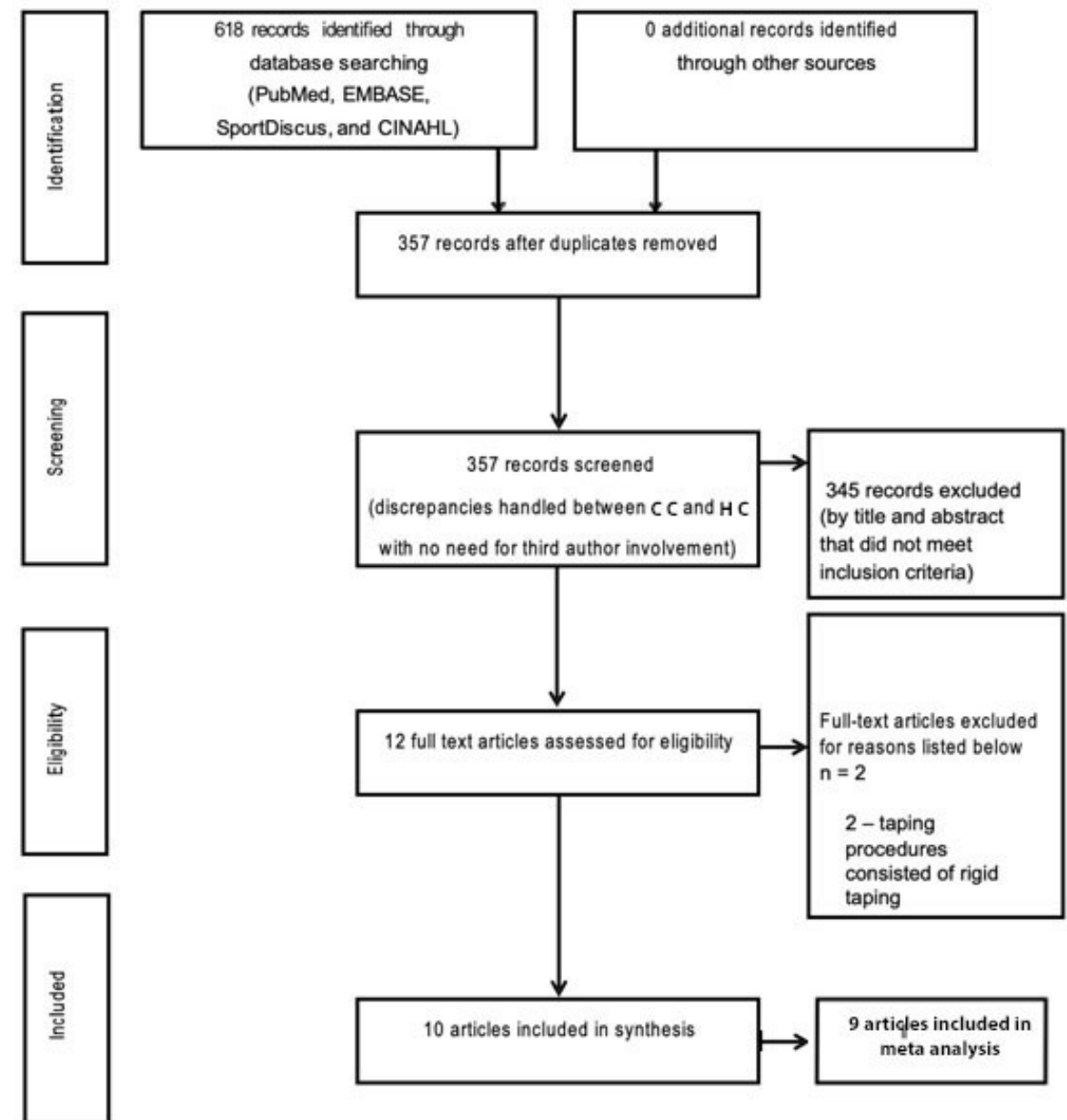

Figure 1: PRISMA flow diagram outlining study selection process

range 2-16). Only one of the 10 studies retained for analysis contained elements for each item resulting in a score of 16. ${ }^{42}$ There was one study that demonstrated a low quality and received a total score of $2 .{ }^{41}$ The remaining studies received a total score of 10 or greater. ${ }^{22,23,25,43-47}$ Although there are no validated cut points for what constitutes a good, fair, or poor score for methodological quality as identified by the modified Downs and Black Checklist, most of the retained studies received a score greater than half of the total possible score. Item 7 (Were the main outcome measures used accurate (valid and reliable)? Accuracy not reported but method clearly described) was scored as yes in only two of the retained studies. ${ }^{42,46}$

\section{TREATMENT FIDELITY}

Treatment fidelity assessments produced a wide value range (Table 2). Out of a maximum of 11 items and 22 total points, the average items identified were $7 \pm 1.9$ (median 7.5, range 3-9) and the total score average was $9.4 \pm 3.0$ (median 10.5, range 3-13). No individual study had all of the items represented; items 3 (If more than one intervention is described, are they all described equally well?) and 6 (Characteristics to be sought and avoided by treatment provider are addressed a priori, make some mention of credentials) were identified in all studies and item 13 (Were non-specific treatment effects evaluated) was not identified in any of the studies. While no validated cut-off exists for this tool, using our criteria, two studies ${ }^{25,46}$ had item scores $\geq 6$ and total scores $\geq 12$; these studies were independently identified as good treatment fidelity.

\section{META-ANALYSIS}

Of ten studies retained in this systematic review, ${ }^{22,23,25,41-47}$ all but one ${ }^{41}$ provided sufficient data to be included and pooled in the meta-analysis. One study 41 did not report standard deviation information for baseline and post-intervention assessments and was excluded from the meta-analysis. Figures 2-4 represent the meta-analyses for pain, disability, and ROM outcomes, respectively.

Pain meta-analysis. Seven studies ${ }^{25,42-47}$ investigating pain outcomes compared KT with exercise to other interventions. One study compared the addition of KT and exercise to non-steroidal anti-inflammatory drugs (NSAIDs) ${ }^{45}$ vs. a comparator treatment that did not include KT. One study ${ }^{42}$ utilized the NPRS while the remaining studies $^{25,43-47}$ utilized the VAS. Five subgroups were analyzed depending on the intervention comparisons. The overall meta-analysis for comparing KT to no KT or sham KT for pain resulted in a small effect size in favor of the experimental group, but the difference was not statistically sig- 
nificant $(\mathrm{SMD}=-0.16 ; 95 \% \mathrm{CI},-0.40-0.08)$. Significant heterogeneity was present among the included studies (homogeneity statistic $Q=42.92 ; \mathrm{df}=14 ; p<0.001$ ), indicating that the variability in the SMD values were greater than that expected by sampling error alone (Figure 2). 


\section{Table 1: Modified Downs and Black Risk of Bias Assessment}

\begin{tabular}{|c|c|c|c|c|c|c|c|c|c|c|c|c|c|c|c|c|}
\hline Authors & 1 & 2 & 3 & 4 & 5 & 6 & 7 & 8 & 9 & 10 & 11 & 12 & 13 & 14 & 15 & Total \\
\hline Devereaux et al 2015 & $Y$ & $\mathrm{Y}$ & $Y$ & $\mathrm{Y}$ & $\mathrm{Y}$ & $\mathrm{Y}$ & $\mathrm{Y}$ & Y & $\mathrm{Y}$ & $\mathrm{Y}$ & $\mathrm{Y}$ & $\mathrm{Y}$ & Y & Y & Y & 16 \\
\hline Djordjevic et al 2012 & $\mathrm{Y}$ & $\mathrm{Y}$ & $\mathrm{Y}$ & $\mathrm{Y}$ & $\mathrm{Y}$ & $\mathrm{Y}$ & $\mathrm{U}$ & $\mathrm{Y}$ & $\mathrm{Y}$ & $\mathrm{Y}$ & $\mathrm{Y}$ & Y & Y & $\mathrm{N}$ & $\mathrm{Y}$ & 14 \\
\hline Gandhi et al 2016 & $\mathrm{Y}$ & $\mathrm{Y}$ & $\mathrm{N}$ & $\mathrm{N}$ & $\mathrm{N}$ & $\mathrm{N}$ & $\mathrm{N}$ & $U$ & $\mathrm{~N}$ & $\mathrm{~N}$ & $N$ & $\mathrm{~N}$ & $\mathrm{~N}$ & $\mathrm{~N}$ & $\mathrm{~N}$ & 2 \\
\hline Goksu et al 2016 & $\mathrm{Y}$ & $\mathrm{Y}$ & $\mathrm{Y}$ & $\mathrm{Y}$ & $\mathrm{Y}$ & $\mathrm{Y}$ & $U$ & $\mathrm{Y}$ & $\mathrm{Y}$ & $\mathrm{Y}$ & $\mathrm{Y}$ & $\mathrm{Y}$ & $\mathrm{Y}$ & $\mathrm{Y}$ & $\mathrm{Y}$ & 15 \\
\hline Kaya et al 2014 & $\mathrm{Y}$ & $\mathrm{Y}$ & $\mathrm{Y}$ & $\mathrm{Y}$ & Y & $\mathrm{Y}$ & $\mathrm{U}$ & N & $\mathrm{Y}$ & $\mathrm{Y}$ & $\mathrm{Y}$ & $\mathrm{Y}$ & $\mathrm{N}$ & $\mathrm{Y}$ & $\mathrm{Y}$ & 13 \\
\hline Onat et al 2016 & $\mathrm{Y}$ & $\mathrm{Y}$ & $\mathrm{Y}$ & $u$ & Y & $\mathrm{Y}$ & $u$ & $u$ & $\mathrm{Y}$ & $\mathrm{Y}$ & $\mathrm{Y}$ & $\mathrm{Y}$ & $\mathrm{N}$ & $\mathrm{N}$ & $\mathrm{N}$ & 10 \\
\hline Pekyavas \& Baltaci 2016 & $\mathrm{Y}$ & $\mathrm{Y}$ & $\mathrm{Y}$ & $\mathrm{Y}$ & $Y$ & $\mathrm{Y}$ & U & $\mathrm{Y}$ & $\mathrm{Y}$ & $\mathrm{Y}$ & $Y$ & $u$ & $\mathrm{U}$ & $\mathrm{Y}$ & $\mathrm{Y}$ & 12 \\
\hline Simsek et al 2013 & $\mathrm{Y}$ & $\mathrm{Y}$ & Y & $u$ & Y & $\mathrm{Y}$ & $U$ & $\mathrm{Y}$ & $\mathrm{Y}$ & $\mathrm{Y}$ & $\mathrm{Y}$ & U & $\mathrm{Y}$ & $\mathrm{Y}$ & $\mathrm{Y}$ & 12 \\
\hline Subasi et al 2016 & $\mathrm{Y}$ & $\mathrm{N}$ & $\mathrm{Y}$ & $\mathrm{Y}$ & $\mathrm{N}$ & $\mathrm{Y}$ & Y & $\mathrm{Y}$ & $\mathrm{Y}$ & $\mathrm{Y}$ & $\mathrm{Y}$ & $\mathrm{Y}$ & $\mathrm{N}$ & $\mathrm{N}$ & $\mathrm{N}$ & 11 \\
\hline Tantawy \& Kamel 2016 & $\mathrm{Y}$ & $\mathrm{Y}$ & $\mathrm{N}$ & $N$ & $Y$ & $Y$ & $U$ & $\mathrm{Y}$ & $Y$ & $\mathrm{Y}$ & $Y$ & $\mathrm{Y}$ & $\mathrm{N}$ & $\mathrm{N}$ & $\mathrm{N}$ & 10 \\
\hline
\end{tabular}

$1=$ yes, $0=$ no/unsure, (item 12 scoring only; $2=y e s, 1=$ unsure, $0=$ no). Criteria 1-15, per the Modified Downes and Black Checklist. 
Table 2: Fidelity Scoring

\begin{tabular}{|l|c|c|c|c|c|c|c|c|c|c|c|c|c|}
\hline Author & $\mathbf{1}$ & $\mathbf{2}$ & $\mathbf{3}$ & $\mathbf{4}$ & $\mathbf{5}$ & $\mathbf{6}$ & $\mathbf{7}$ & $\mathbf{8}$ & $\mathbf{9}$ & $\mathbf{1 0}$ & $\mathbf{1 1}$ & Summary & $\begin{array}{l}\text { Total } \\
\text { Score }\end{array}$ \\
\hline $\begin{array}{l}\text { Devereaux } \\
\text { et al 2015 }\end{array}$ & 2 & 1 & 1 & 1 & 1 & 1 & 2 & 0 & 0 & 0 & 0 & 7 \\
\hline $\begin{array}{l}\text { Djordjevic et } \\
\text { al 2012 }\end{array}$ & 2 & 2 & 2 & 0 & 0 & 2 & 2 & 1 & 0 & 0 & 0 & 6 & 11 \\
\hline $\begin{array}{l}\text { Gandhi et al } \\
2016\end{array}$ & 0 & 0 & 1 & 0 & 0 & 1 & 1 & 0 & 0 & 0 & 0 & 3 & 3 \\
\hline $\begin{array}{l}\text { Goksu et al } \\
2016\end{array}$ & 1 & 1 & 1 & 0 & 0 & 1 & 2 & 0 & 0 & 0 & 0 & 5 & 6 \\
\hline $\begin{array}{l}\text { Kaya et al } \\
2014\end{array}$ & 1 & 1 & 2 & 0 & 1 & 1 & 2 & 1 & 1 & 1 & 0 & 9 & 11 \\
\hline $\begin{array}{l}\text { Onat et al } \\
2016\end{array}$ & 1 & 1 & 2 & 1 & 1 & 2 & 0 & 1 & 1 & 1 & 0 & 9 & 11 \\
\hline $\begin{array}{l}\text { Pekyavas \& } \\
\text { Baltaci 2016 }\end{array}$ & 1 & 1 & 1 & 1 & 1 & 1 & 2 & 0 & 0 & 0 & 0 & 7 \\
\hline $\begin{array}{l}\text { Simsek et al } \\
2013\end{array}$ & 1 & 2 & 2 & 0 & 1 & 1 & 1 & 0 & 1 & 1 & 0 & 8 \\
\hline $\begin{array}{l}\text { Subasi et al } \\
2016\end{array}$ & 2 & 2 & 1 & 1 & 1 & 1 & 2 & 0 & 2 & 0 & 0 & 8 \\
\hline $\begin{array}{l}\text { Tantawy \& } \\
\text { Kamel 2016 }\end{array}$ & 2 & 2 & 2 & 0 & 1 & 1 & 2 & 0 & 2 & 1 & 0 & 8 \\
\hline
\end{tabular}

0 - not present; 1 - minimally described; 2 - more than minimally described

Key to Fidelity criteria:

1. Is there information about treatment dose in the intervention condition?

2. Is there information about treatment dose in the control/comparison condition?

3. If more than one intervention is described, are they all described equally well?

4. Method to ensure dose is equivalent between conditions

5. Method to ensure dose is equivalent within conditions

6. Characteristics to be sought and avoided by treatment provider are addressed a priori, make some mention of credentials

7. Is there mention of a theoretical model or clinical guidelines upon which the intervention is based?

8. Do the authors indicate how providers were trained? Do the authors indicate that provider training was standardized?

9. Was there a method to ensure that the content of the intervention was being delivered as specified?

10. Was there a method to ensure that the dose of the intervention was being delivered as specified?

11. Were non-specific treatment effects evaluated

Disability meta-analysis. Seven studies $23,25,42-44,46,47$ assessing disability were included that compared KT in addition to exercise (as well as manual therapy and highintensity laser therapy in a single study ${ }^{23}$ ) versus a comparator treatment that did not include KT. Two studies ${ }^{42,47}$ utilized the Constant score, four studies $23,25,46$ utilized the SPADI, and two studies 44,47 utilized the DASH. The overall meta-analysis for disability and function resulted in a large and statistically significant effect size (SMD $=-1.35 ; 95 \%$ CI, -2.09 to -0.60 ) in favor of the experimental group. The homogeneity statistic $\mathrm{Q}=121.35(\mathrm{df}=9, p<0.001)$ indicated significant heterogeneity among the included studies, and the variability in the SMD values were greater than that expected by sampling error alone (Figure 3).

ROM meta-analysis. Seven studies $22,23,25,43,45-47$ assessing ROM outcomes were included that compared KT in addition to other conservative treatment with comparator treatments that did not include KT. Two studies ${ }^{23,25}$ measured shoulder external rotation (ER), abduction (ABD), and flexion and the remaining studies ${ }^{43,45-47}$ measured shoulder internal rotation (IR), ER, ABD, and flexion. The metaanalysis revealed a large and statistically significant effect size $(\mathrm{SMD}=0.96$; 95\% CI, $0.60-1.33$ ) in favor of the experi- mental group. The homogeneity statistic $Q=303.18(\mathrm{df}=29$, $p<0.001$ ) indicated significant heterogeneity among the included studies, and the variability in the SMD values were greater than that expected by sampling error alone (Figure 4).

\section{DISCUSSION}

The aim of this study was to perform a systematic review and meta-analysis of the current literature to determine the efficacy and treatment fidelity related to the application of KT on the shoulder complex in conjunction with conservative treatment interventions within a symptomatic population. Within this review, KT was paired with various interventions in order to best mirror how KT is currently utilized in the clinical setting and was compared to other standard practices seen in the treatment of patients with shoulder pathology. Essentially, clinical practice often dictates multi-modal approaches to care, thus adding KT to conservative care would offer improved clinical translation.

All but one study ${ }^{41}$ scored greater than or equal to $10 /$ 16 on the modified Downs and Black checklist. The majority of studies included in this systematic review described a 


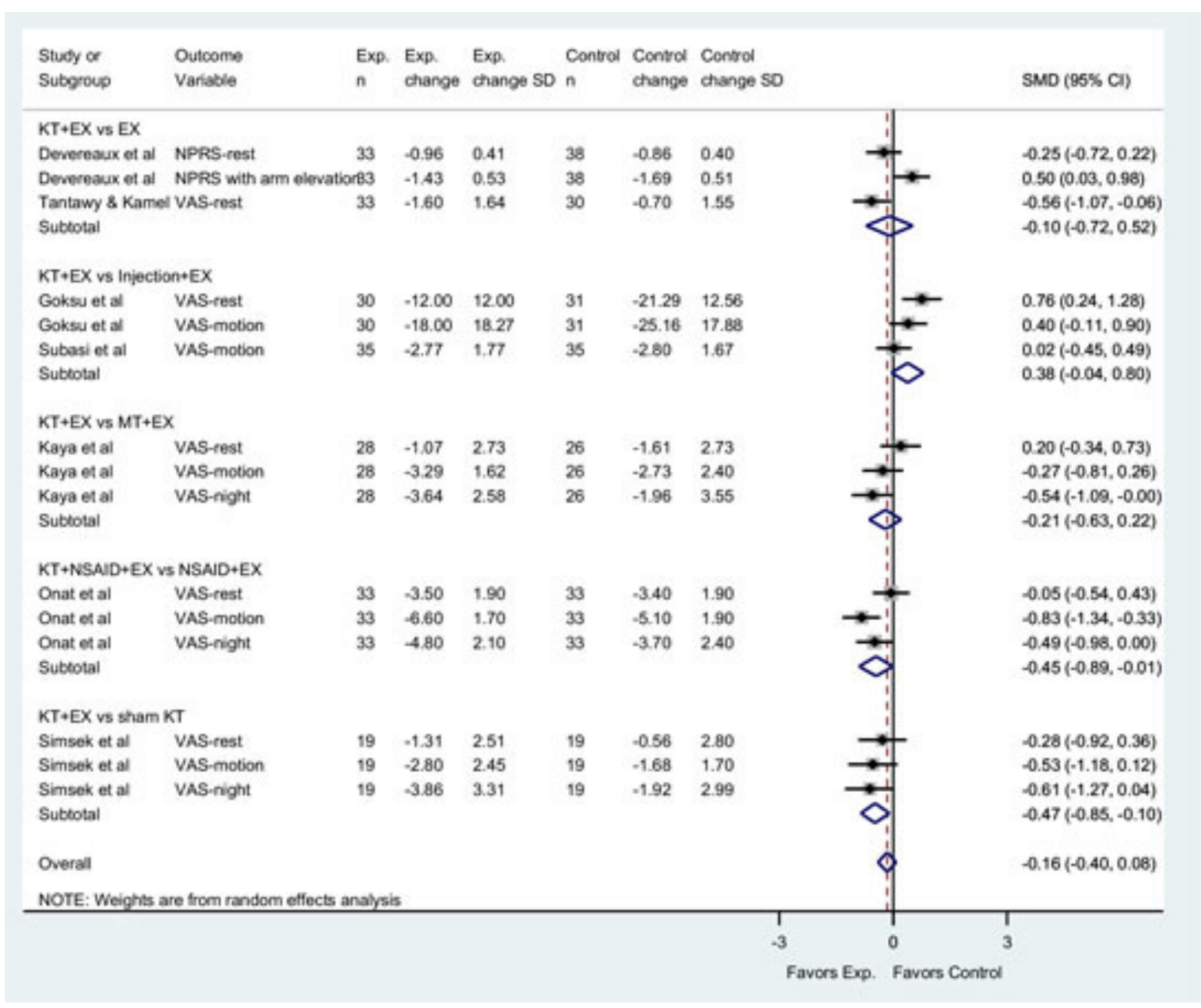

\section{Figure 2: Meta-analysis of kinesiology taping for pain, subgrouped by comparison type}

$\mathrm{KT}=$ kinesiology taping, $\mathrm{EX}=$ exercise, $\mathrm{MT}=$ manual therapy, NSAID= nonsteroidal anti-inflammatory, NPRS= numeric pain rating scale (range $0-10)$, VAS= visual analog scale (0mm = no pain; $100 \mathrm{~mm}=$ worst pain)

patient population similar to that which is typically seen in the clinical setting with their characteristics clearly described, adding to the clinical utility of this review. All studies utilized a comparison group, which allowed comparison of kinesiology taping to commonly-used intervention strategies such as therapeutic exercise, $22,23,25,42-45,47$ manual therapy techniques, ${ }^{22,41,44}$ injections, ${ }^{43,46}$ and NSAIDs. ${ }^{45}$ These studies appeared to demonstrate clinical utility and ease of transference to practice; however, there appears to be lower methodological quality and questionable validity and reliability of outcomes measured. Only two studies $^{42,46}$ clearly described the accuracy and reliability of the outcomes used; four studies ${ }^{22,42,43,47}$ clearly demonstrated appropriate adjustment for confounding variables; and only half of the studies $22,23,42-44,47$ had sufficient power to determine a clinically important effect. While the populations and treatments resemble those commonly seen in the clinic, the included studies may lack the internal validity needed to accurately assess the use of KT in place of interventions that are already supported by literature and currently utilized in practice.

Attention to the critical aspects of treatment fidelity underpins the validity of clinical research and is a prerequisite for the implementation of evidence-based clinical practice. Despite the importance of treatment fidelity, it is an often- neglected component of intervention. To our knowledge, this is the first study to evaluate treatment fidelity of clinical KT interventions. Only two studies ${ }^{25,46}$ were identified to have good treatment fidelity based on the criteria set forth. ${ }^{38}$ These results suggest the majority of studies included in this review could have been influenced by factors such as a lack of researcher training, lack of adherence to protocols, or interventions not performed as specified. Lack of treatment fidelity could also help to explain why results of studies investigating similar interventions may produce different results. ${ }^{35}$ This is important to note, especially when considering the use of KT in place of a more invasive or higher cost treatment, such as receiving injections or the prescription of NSAIDs. For example, Goksu et al. ${ }^{43}$ reported significant improvements after either injections or KT for shoulder impingement patients, although this study demonstrated a lack of treatment fidelity. In comparison, Subasi et al. ${ }^{46}$ had similar findings but demonstrated increased adherence to items associated with treatment fidelity; therefore, KT may be favored as a less invasive clinical treatment. Treatment fidelity should be considered when comparing outcomes of different studies utilizing similar interventions.

The meta-analysis revealed a large effect size for both disability and ROM favoring the experimental group and a 


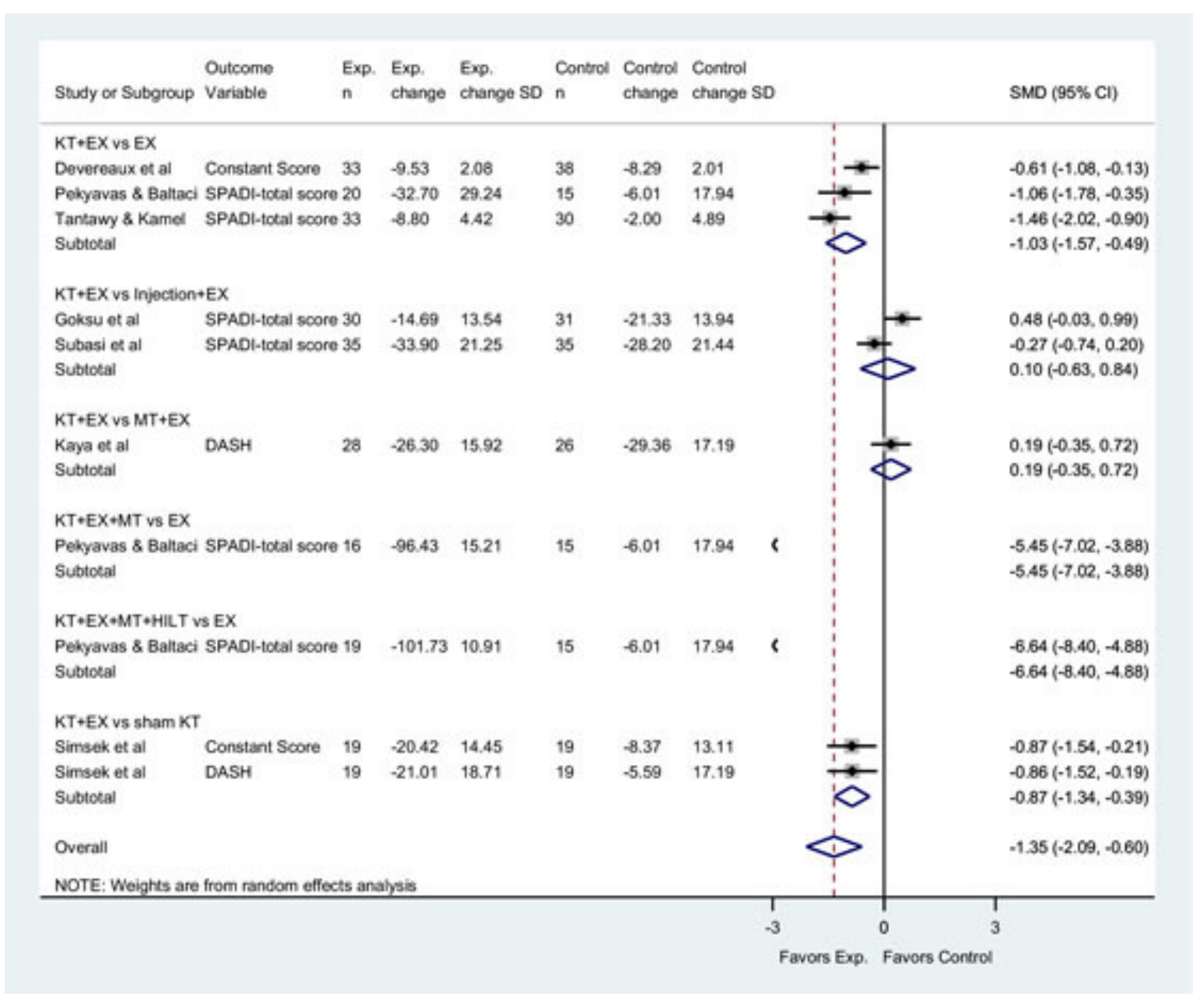

\section{Figure 3: Meta-analysis of kinesiology taping for disability, subgrouped by comparison type}

KT= kinesiology taping, EX= exercise, MT= manual therapy, HILT= high-intensity laser therapy, DASH= Disability of Arm and Shoulder Questionnaire $(0=$ no disability, $100=$ most severe disability), Constant score (the higher the score, the higher the quality of the shoulder function), SPADI= Shoulder Pain and Disability Index (higher scores indicate greater pain and disability). NOTE: the direction of the Constant Score was reversed.

small, statistically insignificant effect size for pain in favor of the experimental group. Although the meta-analysis favors the experimental group for both disability and ROM, these findings must be considered in the presence of both the methodological quality and treatment fidelity of the included studies. Of those studies investigating the effect of KT and other conservative treatment on disability as well as ROM, only two studies ${ }^{25,46}$ had a treatment fidelity score considered to be "good." Furthermore, of the 10 studies retained for analysis, only four $22,25,41,47$ demonstrated a significant between-group improvement when compared to the paired intervention alone considering their chosen outcome measures. One of these studies ${ }^{41}$ had the lowest scores of both methodologic quality and treatment fidelity among those included.

The findings of these studies, even in the context of this review, cannot be interpreted alone without methodological quality and treatment fidelity being considered. When fundamental limitations in treatment fidelity exist such as those found among a majority of the retained studies; lack of reported clinician training, lack of adherence to protocols, and interventions not performed as specified, the validity of the findings may be questioned. Adhering to and appropriately reporting elements related to treatment fi- delity are necessary to maintain the integrity of intervention-based research.

\section{LIMITATIONS}

There are limitations to this study, such as the inclusion of only those studies published in the English language. The authors also did not include a search of the grey literature as the content area being investigated with this type of search would not produce results beyond our current search given the search strategy initially implemented. Methodological quality was assessed utilizing the Modified Downs and Black Assessment Tool, which requires categorization of sub-elements within each study based on the assessor's determination, which could introduce bias. However, this was minimized by having two authors independently score each of the retained studies and discuss any discrepancies. The same limitation may also be considered regarding the assessment of treatment fidelity. Finally, the tool we utilized to evaluate treatment fidelity has not been validated in the modified form. 


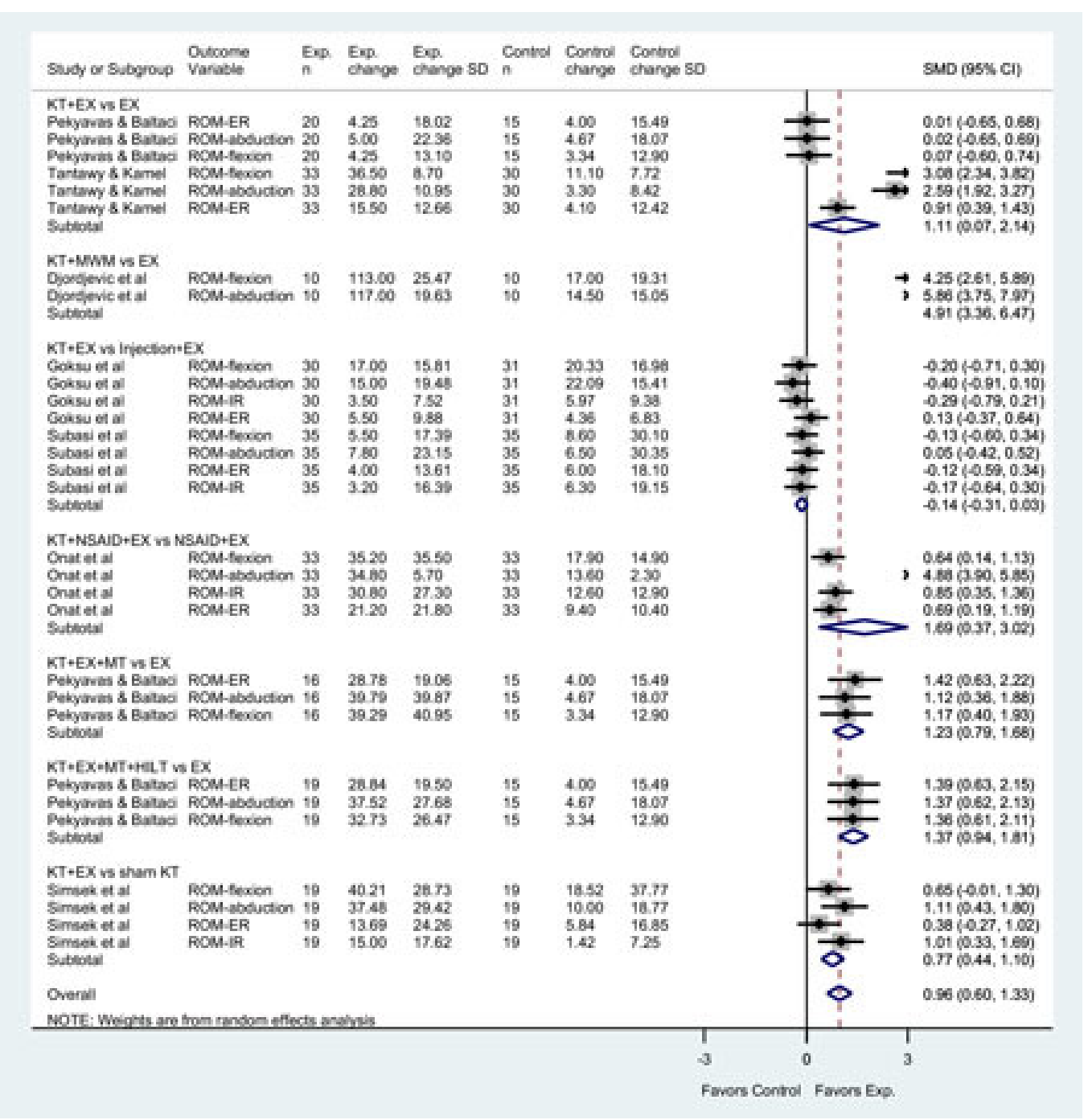

Figure 4: Meta-analysis of kinesiology taping for range of motoin, subgrouped by comparison type

$\mathrm{KT}=$ kinesiology taping, $\mathrm{EX}=$ exercise, $\mathrm{MWM}=$ mobilization with movement, $\mathrm{MT}=$ manual therapy, HILT= high-intensity laser therapy, NSAID= non-steroidal anti-inflammatory drug, $\mathrm{ROM}=$ range of motion, $\mathrm{IR}=$ internal rotation, $\mathrm{ER}=$ external rotation

\section{CONCLUSION}

Although the addition of KT to conservative interventions may demonstrate some efficacy with regard to disability and ROM when compared to conservative interventions alone, these findings must be considered in light of the quality and treatment fidelity associated with these studies. A majority of the identified studies demonstrate fair methodologic quality; however, there were limitations surrounding treatment fidelity. Treatment fidelity is paramount to the implementation of evidence-based practice; thus, limited fidelity may in fact be of greater clinical value than quantitative changes when interpreting the result. Clinicians should consider these findings when determining if KT would be an appropriate adjunct in the treatment of those individuals presenting with shoulder pathology. Fu- ture studies on KT that adhere to the attributes of treatment fidelity may serve to reduce variability of results and provide a more practical outcome that can be replicated in the clinic.

\section{ACKNOWLEDGEMENTS}

Leila Ledbetter

\section{CONFLICTS OF INTERESTS}

The authors report no conflicts of interest.

Submitted: August 06, 2020 CDT, Accepted: November 26, 2020 CDT

This is an open-access article distributed under the terms of the Creative Commons Attribution 4.0 International License (CCBY-NC-SA-4.0). View this license's legal deed at https://creativecommons.org/licenses/by-nc-sa/4.0 and legal code at https://creativecommons.org/licenses/by-nc-sa/4.0/legalcode for more information. 


\section{REFERENCES}

1. Luime JJ, Koes BW, Hendriksen IJ, et al. Prevalence and incidence of shoulder pain in the general population; a systematic review. Scand J Rheumatol. 2004;33(2):73-81.

2. Linsell L, Dawson J, Zondervan K, et al. Prevalence and incidence of adults consulting for shoulder conditions in UK primary care; patterns of diagnosis and referral. Rheumatology (Oxford). 2006;45(2):215-221. doi:10.1093/rheumatology/kei13 9

3. Dean BJ, Gwilym SE, Carr AJ. Why does my shoulder hurt? A review of the neuroanatomical and biochemical basis of shoulder pain. Br J Sports Med. 2013;47(17):1095-1104. doi:10.1136/bjsports-2012-09 1492

4. Lewis J. Frozen shoulder contracture syndrome Aetiology, diagnosis and management. Man Ther. 2015;20(1):2-9. doi:10.1016/j.math.2014.07.006

5. Lewis J. Rotator cuff related shoulder pain: Assessment, management and uncertainties. Man Ther. 2016;23:57-68. doi:10.1016/j.math.2016.03.009

6. Haik MN, Alburquerque-Sendin F, Moreira RF, Pires ED, Camargo PR. Effectiveness of physical therapy treatment of clearly defined subacromial pain: a systematic review of randomised controlled trials. Br J Sports Med. 2016;50(18):1124-1134. doi:1 0.1136/bjsports-2015-095771

7. Louw A, Puentedura EJ, Reese D, Parker P, Miller T, Mintken PE. Immediate Effects of Mirror Therapy in Patients With Shoulder Pain and Decreased Range of Motion. Arch Phys Med Rehabil.

2017;98(10):1941-1947. doi:10.1016/j.apmr.2017.03.0 $\underline{31}$

8. Luque-Suarez A, Navarro-Ledesma S, Petocz P, Hancock MJ, Hush J. Short term effects of kinesiotaping on acromiohumeral distance in asymptomatic subjects: a randomised controlled trial. Man Ther. 2013;18(6):573-577. doi:10.1016/i.math.20 $\underline{13.06 .002}$

9. Van Herzeele M, van Cingel R, Maenhout A, De Mey $\mathrm{K}$, Cools A. Does the application of kinesiotape change scapular kinematics in healthy female handball players? Int J Sports Med. 2013;34(11):950-955. doi:10.1055/s-0033-1334911
10. Gonzalez-Iglesias J, Fernandez-de-Las-Penas C, Cleland JA, Huijbregts P, Del Rosario Gutierrez-Vega $M$. Short-term effects of cervical kinesio taping on pain and cervical range of motion in patients with acute whiplash injury: a randomized clinical trial. $J$ Orthop Sports Phys Ther. 2009;39(7):515-521. doi:10.2 519/jospt.2009.3072

11. Thelen MD, Dauber JA, Stoneman PD. The clinical efficacy of kinesio tape for shoulder pain: a randomized, double-blinded, clinical trial. J Orthop Sports Phys Ther. 2008;38(7):389-395. doi:10.2519/jos pt.2008.2791

12. Alam S, Malhotra D, Munjal J, Chachra A. Immediate effect of Kinesio taping on shoulder muscle strength and range of motion in healthy individuals: A randomised trial. Hong Kong Physiother J. 2015;33(2):80-88. doi:10.1016/i.hkpj.2014.10.004

13. Han JT, Lee JH, Yoon CH. The mechanical effect of kinesiology tape on rounded shoulder posture in seated male workers: a single-blinded randomized controlled pilot study. Physiother Theory Pract. 2015;31(2):120-125. doi:10.3109/09593985.2014.9600 $\underline{54}$

14. Huang YC, Chang KH, Liou TH, Cheng CW, Lin LF, Huang SW. Effects of Kinesio taping for stroke patients with hemiplegic shoulder pain: A doubleblind, randomized, placebo-controlled study. J Rehabil Med. 2017;49(3):208-215. doi:10.2340/165019 $\underline{77-2197}$

15. Aarseth LM, Suprak DN, Chalmers GR, Lyon L, Dahlquist DT. Kinesio Tape and Shoulder-Joint Position Sense. J Athl Train. 2015;50(8):785-791. doi:1 $\underline{0.4085 / 1062-6050-50.7 .03}$

16. Bradley T, Baldwick C, Fischer D, Murrell GA. Effect of taping on the shoulders of Australian football players. Br J Sports Med. 2009;43(10):735-738. doi:10.1136/bjsm.2008.049858

17. Chatterjee S, Hayner KA, Arumugam N, et al. The California Tri-pull Taping Method in the Treatment of Shoulder Subluxation After Stroke: A Randomized Clinical Trial. N Am J Med Sci. 2016;8(4):175-182. do $\mathrm{i}: 10.4103 / 1947-2714.179933$

18. Mostafavifar M, Wertz J, Borchers J. A systematic review of the effectiveness of kinesio taping for musculoskeletal injury. Phys Sportsmed. 2012;40(4):33-40. doi:10.3810/psm.2012.11.1986 
19. Appel C, Perry L, Jones F. Shoulder strapping for stroke-related upper limb dysfunction and shoulder impairments: systematic review. NeuroRehabil. 2014;35(2):191-204. doi:10.3233/nre-141108

20. Desjardins-Charbonneau A, Roy JS, Dionne CE, Desmeules F. The efficacy of taping for rotator cuff tendinopathy: a systematic review and meta-analysis. Int J Sports Phys Ther. 2015;10(4):420-433.

21. Saracoglu I, Emuk Y, Taspinar F. Does taping in addition to physiotherapy improve the outcomes in subacromial impingement syndrome? A systematic review. Physiother Theory Pract. 2018;34(4):251-263. $\underline{\mathrm{d}}$ oi:10.1080/09593985.2017.1400138

22. Djordjevic OC, Vukicevic D, Katunac L, Jovic S. Mobilization With Movement and Kinesiotaping Compared With a Supervised Exercise Program for Painful Shoulder: Results of a Clinical Trial. J Manipulative Physiol Ther. 2012;35(6):454-463. doi:1 0.1016/j.jmpt.2012.07.006

23. Pekyavas NO, Baltaci G. Short-term effects of high-intensity laser therapy, manual therapy, and Kinesio taping in patients with subacromial impingement syndrome. Lasers Med Sci. 2016;31(6):1133-1141. doi:10.1007/s10103-016-196 $\underline{3-2}$

24. Pekyavas NO, Ergun N. Effects of different exercise and kinesiotaping application on pain, flexibility, strength and range of motion in patients with subacromial impingement syndrome. Fizyoterapi Rehabilitasyon. 2014;25(1):S31.

25. Tantawy SA, Kamel DM. The effect of kinesio taping with exercise compared with exercise alone on pain, range of motion, and disability of the shoulder in postmastectomy females: a randomized control trial. J Phys Ther Sci. 2016;28(12):3300-3305. doi:10.1 589/jpts.28.3300

26. Moher D, Liberati A, Tetzlaff J, Altman DG. Preferred reporting items for systematic reviews and meta-analyses: the PRISMA statement. PLoS Med. 2009;6(7):e1000097. doi:10.1371/journal.pmed.10000 $\underline{97}$

27. Higgins JPT, Thomas J, Chandler J, et al., eds. Cochrage Handbook for Systematic Reviews of Interventions. 2nd ed. Chichester: John Wiley \& Sons; 2019.

28. Downs SH, Black N. The feasibility of creating a checklist for the assessment of the methodological quality both of randomised and non-randomised studies of health care interventions. J Epidemiol Community Health. 1998;52(6):377-384.
29. Irving DB, Cook JL, Menz HB. Factors associated with chronic plantar heel pain: a systematic review. $J$ Sci Med Sport. 2006;9(1-2):11-22. doi:10.1016/i.jsam s.2006.02.004

30. Mosler AB, Agricola R, Weir A, Holmich P, Crossley KM. Which factors differentiate athletes with hip/groin pain from those without? A systematic review with meta-analysis. Br J Sports Med. 2015;49(12):810. doi:10.1136/bjsports-2015-094602

31. Munn J, Sullivan SJ, Schneiders AG. Evidence of sensorimotor deficits in functional ankle instability: a systematic review with meta-analysis. J Sci Med Sport. 2010;13(1):2-12. doi:10.1016/j.jsams.2009.03.004

32. Reiman MP, Sylvain J, Loudon JK, Goode A. Return to sport after open and microdiscectomy surgery versus conservative treatment for lumbar disc herniation: a systematic review with meta-analysis. Br J Sports Med. 2016;50(4):221-230. doi:10.1136/bjsp orts-2015-094691

33. Bellg AJ, Borrelli B, Resnick B, et al. Enhancing treatment fidelity in health behavior change studies: best practices and recommendations from the NIH Behavior Change Consortium. Health Psychol. 2004;23(5):443-451. doi:10.1037/0278-6133.23.5.443

34. Carpenter JS, Burns DS, Wu J, et al. Strategies used and data obtained during treatment fidelity monitoring. Nurs Res. 2013;62(1):59-65. doi:10.1097/ NNR.0b013e31827614fd

35. Cook CE, George SZ, Keefe F. Different interventions, same outcomes? Here are four good reasons. Br J Sports Med. 2018;52(15):951-952. doi:1 0.1136/bjsports-2017-098978

36. Borrelli B, Sepinwall D, Ernst D, et al. A new tool to assess treatment fidelity and evaluation of treatment fidelity across 10 years of health behavior research. J Consult Clin Psychol. 2005;73(5):852-860. $\underline{\mathrm{d}}$ oi:10.1037/0022-006x.73.5.852

37. Toomey E, Currie-Murphy L, Matthews J, Hurley DA. Implementation fidelity of physiotherapistdelivered group education and exercise interventions to promote self-management in people with osteoarthritis and chronic low back pain: a rapid review part II. Man Ther. 2015;20(2):287-294. doi:10.1 016/j.math.2014.10.012

38. Salamh P, Cook C, Reiman MP, Sheets C. Treatment effectiveness and fidelity of manual therapy to the knee: A systematic review and metaanalysis. Musculoskeletal Care. 2017;15(3):238-248. do i:10.1002/msc.1166 
39. Borenstein M, Hedges LV, Higgins JP, Rothstein HR. Introduction to Meta-Analysis. West Sussex, UK: John Wiley \& Sons, Ltd.; 2009.

40. Hedges LV, Olkin I. Statistical Methods for MetaAnalysis. Orlando (FL): Academic Press; 1985. doi:1 0.1016/B978-0-08-057065-5.50016-6

41. Gandhi VM, Arun B, Kumar RKP. Effectiveness of myofascial release therapy with shoulder taping on subacromial impingement syndrome in collegiate basket ball players - A quasi experimental pilot study. Bangladesh J Med Sci. 2016;15(3):347-351. doi:10.332 9/bims.v15i3.21292

42. Devereaux M, Velanoski KQ, Pennings A, Elmaraghy A. Short-term effectiveness of precut kinesiology tape versus an nsaid as adjuvant treatment to exercise for subacromial impingement: A randomized controlled trial. Clin J Sport Med. 2016;26(1):24-32. doi:10.1097/JSM.000000000000018 7

43. Goksu H, Tuncay F, Borman P. The comparative efficacy of kinesio taping and local injection therapy in patients with subacromial impingement syndrome. Acta Orthop Traumatol Turc. 2016;50(5):483-488. do i:10.1016/i.aott.2016.08.015
44. Kaya DO, Baltaci G, Toprak U, Atay AO. The clinical and sonographic effects of kinesiotaping and exercise in comparison with manual therapy and exercise for patients with subacromial impingement syndrome: A preliminary trial. J Manipulative Physiol Ther. 2014;37(6):422-432. doi:10.1016/i.jmpt.2014.0 3.004

45. Onat ŞŞ, Biçer S, Şahin Z, Türkyılmaz AK, Kara M, Demir SÖ. Effectiveness of Kinesiotaping and Subacromial Corticosteroid Injection in Shoulder Impingement Syndrome. Am J Phys Med Rehabil. 2016;95(8):553-560.

46. Subasi V, Cakir T, Arica Z, et al. Comparison of efficacy of kinesiological taping and subacromial injection therapy in subacromial impingement syndrome. Clin Rheumatol. 2016;35(3):741-746. doi:1 0.1007/s10067-014-2824-7

47. Şimşek HH, Balki S, Keklik SS, Ozturk H, Elden H. Does Kinesio taping in addition to exercise therapy improve the outcomes in subacromial impingement syndrome? A randomized, double-blind, controlled clinical trial. Acta Ortho Traumatol Turcica. 2013;47(2):104-110. doi:10.3944/AOTT.2013.2782 


\section{SUPPLEMENTARY MATERIALS}

\section{Supplement 1}

Download: https://ijspt.scholasticahq.com/article/24251-the-efficacy-and-treatment-fidelity-of-kinesiology-taping-inconjunction-with-conservative-treatment-interventions-among-individuals-with-shoulder-pai/attachment/61424.pdf

\section{Appendix A}

Download: https://ijspt.scholasticahq.com/article/24251-the-efficacy-and-treatment-fidelity-of-kinesiology-taping-inconjunction-with-conservative-treatment-interventions-among-individuals-with-shoulder-pai/attachment/ 61425.docx 